\title{
Autophagy promotes malignant migration and invasion via miR-224-5p/BCL2 in pancreatic mucinous cystadenocarcinoma MCC1 cells
}

\author{
CHENGTAO GUO $^{1 *}$, XIAOBO PENG $^{1 *}$, LELE SONG $^{2}$, MINGZHEN YING $^{1}$, YANJUN WU $^{2}$, \\ RENXU CHANG $^{2}$, JIE LI ${ }^{1}$, DAN FENG ${ }^{1}$, LIXING ZHAN ${ }^{2}$ and XIANBAO ZHAN ${ }^{1}$ \\ ${ }^{1}$ Department of Oncology, Changhai Hospital, The Second Military Medical University, Shanghai 200433; \\ ${ }^{2}$ Key Laboratory of Food Safety Research, Institute for Nutritional Sciences, Shanghai Institutes for \\ Biological Sciences, Chinese Academy of Sciences, Shanghai 200031, P.R. China
}

Received February 27, 2019; Accepted October 10, 2019

DOI: $10.3892 / \mathrm{ol} .2020 .12139$

\begin{abstract}
The prognosis of invasive pancreatic mucinous cystadenocarcinoma (MCC) is poor, and the molecular mechanism underlying its development remains unclear. The present study aimed to explore the potential role of autophagy in pancreatic MCC. The results demonstrated an increase in autophagy signaling in pancreatic MCC tissues and the MCC1 cell line compared with adjacent tissues and normal human pancreatic ductal epithelium (HPDE) cells. In addition, abnormal autophagy activation facilitated the migration and invasion of MCC1 cells. MicroRNA (miR)-224-5p expression levels were significantly higher in MCC1 cells compared with those in HPDE cells. Treatment with rapamycin further demonstrated that high levels of autophagy elevated miR-224-5p expression in MCC1 cells in a time-dependent manner. BCL2 was identified as a downstream target gene of miR-224-5p, which
\end{abstract}

Correspondence to: Professor Xianbao Zhan, Department of Oncology, Changhai Hospital, The Second Military Medical University, 168 Changhai Road, Yangpu, Shanghai 200433, P.R. China

E-mail: zhanxianbao@126.com

Professor Lixing Zhan, Key Laboratory of Food Safety Research, Institute for Nutritional Sciences, Shanghai Institutes for Biological Sciences, Chinese Academy of Sciences, 320 Yueyang Road, Xuhui, Shanghai 200031, P.R. China

E-mail: 1xzhan@sibs.ac.cn

${ }^{*}$ Contributed equally

Abbreviations: MCNs, mucinous cystic neoplasms; MCC, mucinous cystadenocarcinoma; mTOR, mammalian target of rapamycin; miRNA, microRNA; IHC, immunohistochemistry; RT-qPCR, reverse transcription-quantitative PCR; HPDE, human pancreatic epithelial cell; DMSO, dimethyl sulfoxide

Key words: autophagy, mucinous cystadenocarcinoma, MCC1, metastasis, microRNA-224-5p, Bcl2 binds to the 3'-untranslated region of BCL2. In addition, the results of the present study demonstrated that BCL2 knockdown reversed the inhibition of autophagy mediated by the miR-224-5p inhibitor. To the best of our knowledge, this is the first study to evaluate the role of autophagy in pancreatic MCC. Thus, these results suggested that autophagy may be hyperactivated in pancreatic MCC. In addition, the present study identified a positive feedback loop between autophagy signaling and miR-224-5p, which may promote the aggressive migration and invasion of MCC1. These results may provide a new insight into the relationship between autophagy and tumor metastasis in pancreatic MCC.

\section{Introduction}

Mucinous cystic neoplasms (MCNs) are one of the most common types of cystic neoplasms of the pancreas that specifically occur in the pancreatic body or tail of perimenopausal women (1). With the continuous application and progress of cross-sectional imaging technology, the number of patients diagnosed with pancreatic MCNs has increased over time (2). In the 1970s, Compagno and Oertel (3) proposed that pancreatic MCNs eventually become malignant as the disease progresses. A multicenter retrospective analysis reported that MCC or high-grade dysplasia is present in $14.9 \%$ of resected pancreatic MCNs (4). The 5-year survival rate of invasive pancreatic MCC is $<20 \%$, which is similar to that of ductal adenocarcinoma of the pancreas (5). Due to its malignant potential, a number of studies have focused on pancreatic MCNs (5-7). However, few studies have explored the molecular mechanism underlying the development of invasive pancreatic MCC.

Autophagy, which is a highly conserved catabolic process, is critical for cellular homeostasis under normal physiological conditions, as well as for cell viability under stress. The mammalian target of rapamycin (mTOR)/AMP-activated protein kinase pathway serves an essential role in regulating this process $(8,9)$. Under physiological conditions, autophagy is considered to inhibit the malignant transformation of cells by maintaining cell homeostasis and normal metabolism (10). With the exception of autophagy-mediated suppression in 
Table I. Clinicopathological data of four cases of pancreatic mucinous cystadenocarcinoma.

\begin{tabular}{|c|c|c|c|c|c|}
\hline $\begin{array}{l}\text { Patient } \\
\text { number }\end{array}$ & Sex & $\begin{array}{c}\text { Age } \\
\text { (years) }\end{array}$ & Pathological diagnosis & Tumor location & $\begin{array}{l}\text { Lymphatic } \\
\text { metastasis }\end{array}$ \\
\hline 1 & Female & 76 & $\begin{array}{l}\text { Mucinous cystadenoma of the pancreas with high-grade } \\
\text { intraepithelial neoplasia, carcinogenesis and local } \\
\text { infiltration of the stroma }\end{array}$ & Pancreatic tail & $0 / 1$ \\
\hline 2 & Female & 47 & Invasive mucinous cystadenocarcinoma of the pancreas & Pancreatic body and tail & $0 / 1$ \\
\hline 3 & Female & 47 & Invasive mucinous cystadenocarcinoma of the pancreas & Pancreatic body and tail & \\
\hline 4 & Female & 62 & $\begin{array}{l}\text { Pancreatic mucinous cystic tumor with invasive carcinoma } \\
\text { (mucinous cystadenocarcinoma) }\end{array}$ & Pancreatic body and tail & $0 / 7$ \\
\hline
\end{tabular}

malignant transformation of normal cells, autophagy can facilitate tumorigenesis and generate resistance to anti-tumor therapy under adverse microenvironment conditions (10). Previous studies have suggested that autophagy regulates tumor metastasis by affecting local adhesion, anti-anoikis and epithelial-mesenchymal transition (EMT) (11). However, the function of autophagy in pancreatic MCC metastasis remains unclear.

Considering the poor survival associated with aggressive malignancy, it is necessary to study the potential regulators of pancreatic MCC metastasis. Based on the widespread involvement of autophagy in tumor development, its potential for targeted therapy has been suggested. The purpose of this study was to investigate the effect of autophagy on the malignant migration and invasion of $\mathrm{MCC} 1$ cells.

\section{Materials and methods}

Clinical samples. A total of 4 paired paraffin-embedded pancreatic mucinous cystadenocarcinoma samples and matched adjacent tissues were obtained retrospectively from the Department of Pathology, Changhai Hospital (Shanghai, China) (Table I). The distance between the tumor and the adjacent tissue sampling point site was $15 \mathrm{~mm}$. All four patients were female, with a mean age of 58 years, ranging from 47 to 76 years. Tissue samples were rare and all available samples were used for this study. Informed consent was obtained from the subjects prior to the collection of the specimens.

Cell culture and reagents. Human pancreatic ductal epithelial (HPDE) cells were obtained from the Type Culture Collection of the Chinese Academy of Sciences. The cells were cultured with DMEM (Thermo Fisher Scientific, Inc.) supplemented with $10 \%$ fetal bovine serum (HyClone; GE Healthcare Life Sciences) and 1\% penicillin/streptomycin (Thermo Fisher Scientific, Inc.). MCC1 cells were kindly provided by Professor Claudio Sorio from the University of Verona, Italy. The cells were incubated in RPMI-1640 medium (HyClone; GE Healthcare Life Sciences) supplemented with $10 \%$ fetal bovine serum (HyClone; Cyvita), $2 \mathrm{mM}$ glutamine, $80 \mu \mathrm{g} / \mathrm{ml}$ gentamicin sulfate and $2.5 \mu \mathrm{g} / \mathrm{ml}$ amphotericin B (Sigma-Aldrich; Merck KGaA). All the cells were incubated at $37^{\circ} \mathrm{C}$ in a humidified atmosphere with $5 \% \mathrm{CO}_{2}$. Rapamycin and Chloroquine (CQ) were obtained from Sigma-Aldrich; Merck KGaA.
Transwell migration and invasion assays. The Transwell migration assay was performed in 24-well inserts with $8-\mu \mathrm{m}$ pores (Corning, Inc.), whereas the invasion assay was performed in 24 -well inserts with $8-\mu \mathrm{m}$ pores coated with Matrigel (Corning, Inc.) according to the manufacturer's protocols. Cells $\left(8 \times 10^{4}\right.$ cells $/ 200 \mu 1$ of RPMI- 1640 medium containing $0.2 \%$ bovine serum albumin (Sigma-Aldrich; Merck $\mathrm{KGaA}$ ), $2 \mathrm{mM}$ glutamine (Thermo Fisher Scientific, Inc.), $80 \mu \mathrm{g} / \mathrm{ml}$ gentamicin sulfate (Sigma-Aldrich; Merck KGaA) and $2.5 \mu \mathrm{g} / \mathrm{ml}$ amphotericin B (Sigma-Aldrich; Merck KGaA) per insert were seeded into the upper chamber; the bottom side was filled with culture RPMI-1640 medium containing $10 \%$ foetal bovine serum, $2 \mathrm{mM}$ glutamine, $80 \mu \mathrm{g} / \mathrm{ml}$ gentamicin sulfate and $2.5 \mu \mathrm{g} / \mathrm{ml}$ amphotericin B. Following incubation for $6 \mathrm{~h}$, the inserts from the migration and invasion assays were fixed with $4 \%$ paraformaldehyde for $30 \mathrm{~min}$ at room temperature $\left(25^{\circ} \mathrm{C}\right)$ and stained with $0.1 \%$ crystal violet solution for $30 \mathrm{~min}$ at room temperature. Non-migrated cells were removed from the upper membrane using a cotton swab; the migrated cells on the lower membrane were dried and imaged using an Olympus IX51 microscope (Olympus Corporation). The cells were counted using ImageJ software (version 1.44p; National Institutes of Health).

Small interfering (si)RNA and cell transfection. siRNA against BCL2 (siBCL2) was purchased from Guangzhou RiboBio Co., Ltd. The target sequence of BCL2 was as follows: 5'-GGA GAACAGGGTACGATAA-3'. The sequences of the negative control primers were forward, 5'-GGCUCUAGAAAAGCC UAUGCdTdT-3' and reverse, 3'-dTdTCCGAGAUCUUUUCG GAUACG-5'. The plasmids and siRNAs were transfected in miR-224-5p inhibitor and negative control MCC1 cells $\left(5 \times 10^{4}\right)$ using Lipofectamine ${ }^{\circledR} 2000$ reagent (Thermo Fisher Scientific, Inc.) according to the manufacturer's protocol at room temperature for $25 \mathrm{~min}$. The ratio of plasmid or siRNA to lipo is $1 \mu \mathrm{g}$ : $2 \mu \mathrm{l}$. Cells were harvested $48 \mathrm{~h}$ after transfection. Cells were harvested $48 \mathrm{~h}$ after transfection.

Protein extraction and western blot assays. Experimental cells (HPDE and MCC1 cells treated with NCm, miR-224, $\mathrm{NCi}$, inhibitor-224) and $\mathrm{MCC} 1$ treated with rapamycin were lysed with RIPA buffer (Cell Signaling Technology, Inc.) containing complete protease inhibitor cocktail (Roche Diagnostics), phosphatase inhibitors (Roche Diagnostics), $5 \mathrm{mM}$ dithiothreitol (Sigma-Aldrich; Merck KGaA) and 
Table II. Primer sequences of primers used in RT-qPCR.

\begin{tabular}{|c|c|}
\hline Primers & Sequence $\left(5^{\prime} \rightarrow 3^{\prime}\right)$ \\
\hline miR-224-5p & $\begin{array}{l}\text { F: CTCAACTGGTGTCGTGGAGTCGGC } \\
\text { AATTCAGTTGAGCAAGGCAA } \\
\text { R: ACACTCCAGCTGGGCAAGTCACTA } \\
\text { GTGGT }\end{array}$ \\
\hline U6 & $\begin{array}{l}\text { F: CTCGCTTCGGCAGCACA } \\
\text { R: AACGCTTCACGAATTTGCGT }\end{array}$ \\
\hline BCL2 & $\begin{array}{l}\text { F: GGTGGGGTCATGTGTGTGG } \\
\text { R: CGGTTCAGGTACTCAGTCATCC }\end{array}$ \\
\hline $18 \mathrm{~S}$ & $\begin{array}{l}\text { F: ACCGCAGCTAGGAATAATGGA } \\
\text { R: CAAATGCTTTCGCTCTGGTC }\end{array}$ \\
\hline
\end{tabular}

F, forward; R, reverse.

$1 \mathrm{mM}$ phenylmethylsulfonyl fluoride (Sigma-Aldrich; Merck KGaA) and incubated on ice for $30 \mathrm{~min}$. The cell lysate was centrifuged at $12,000 \mathrm{x} \mathrm{g}$ for $10 \mathrm{~min}$ at $4^{\circ} \mathrm{C}$. The supernatant was collected and protein concentrations were determined using the BCA protein assay kit (Thermo Fisher Scientific, Inc.) according to the manufacturer's instructions. Western blot assays were performed as previously described (12). The following antibodies were used: anti-GAPDH (cat. no. M2006M, 1:5,000 dilution) and anti- $\beta$-actin (cat. no. M2001M, 1:5,000 dilution) (both from Abmart Pharmaceutical Technology Co., Ltd.), anti-P62 (cat. no. 5114T, 1:2,000 dilution) and horseradish peroxidase-conjugated secondary antibody (cat. no. 7074S, 1:2,000 dilution) (both from Cell Signaling Technology, Inc.), anti-LC3 (cat. no. L7543, 1:2,000 dilution; Sigma-Aldrich; Merck KGaA) and anti-BCL2 (cat. no. L5034901, 1:2,000 dilution; BD Biosciences). ImageJ (version 1.44p; National Institutes of Health) was used for densitometry analysis.

$R N A$ extraction and reverse transcription-quantitative PCR $(R T-q P C R)$. Total RNA was isolated using TRIzol $^{\circledR}$ reagent (Invitrogen; Thermo Fisher Scientific, Inc.), cDNA was then generated by reverse transcription of aliquots of RNA using the Takara PrimeScript RT Reagent kit (Takara Bio, Inc.) according to the manufacturer's instructions. The resulting cDNA was used for qPCR with SYBR ${ }^{\circledR}$ Premix Ex Taq ${ }^{\text {TM }}$ kit (Takara Bio, Inc.) in a StepOne Real-Time PCR Detection System (Thermo Fisher Scientific, Inc.) (13). For micro (mi)RNA detection, the total RNA was extracted from cells as aforementioned, and then miRNA was reverse transcribed and amplified using a Takara PrimeScript RT Reagent kit according to the manufacturer's instructions. The amplification reactions were performed in triplicate in a 96-well plate using the following thermocycling conditions: $5 \mathrm{~min}$ at $95^{\circ} \mathrm{C}$, followed by 40 cycles of $10 \mathrm{sec}$ at $95^{\circ} \mathrm{C}$ and $30 \mathrm{sec}$ at $60^{\circ} \mathrm{C}$. The Cq values were calculated using ABI Sequence Detection System software (version 2.1; Thermo Fisher Scientific, Inc.). The relative fold-change of each miRNA was calculated using the comparative $2^{-\Delta \Delta \mathrm{C} q}$ method (14). The primers used for qPCR are presented in Table II. The quantitative miRNA data were normalized to U6 and mRNA expression of BCL2 of $18 \mathrm{~S}$.

Vectors and lentiviral transduction. All recombinant lentiviruses were obtained from Shanghai Genechem Co., Ltd. The packaged lentiviruses contained miR-224 mimic-5p (miR-224), miR-224-5p mimic control (NCm), miR-224-5p inhibitor (inhibitor-224) and miR-224-5p inhibitor control (NCi). All negative controls were empty vectors. The sequences were as follows: Hsa-miR-224-5p-mimic forward, 5'-GAG GATCCCCGGGTACCGGCCAGCTAACCATGGGCCTGC CTC-3' and reverse, 5'-CACACATTCCACAGGCTAGAG GAGAAAGAAGACCTCTTTTC-3'; hsa-miR-224-5p-inhibitor forward, 5'-CAAGTCACTAGTGGTTCCGTT-3' and reverse, 5'-AACGGAACCACTAGTGACTTG-3'. Lentiviral transduction was performed following the manufacturer's protocol. The packaged virus solution was thawed, and the virus stock solution was diluted with the infection medium (MOI) value equal to 10 using fresh medium containing the gene transfection enhancer polyamine. After $72 \mathrm{~h}$ of infection at room temperature, the transduction efficiency was evaluated as the percentage of green fluorescent protein-positive cells observed under an inverted fluorescence microscope (magnification x200; Olympus Corporation). The lentivirus-infected cells were treated with $2 \mu \mathrm{g} / \mathrm{ml}$ puromycin for 1 week, and the cells resistant to puromycin were selected.

Immunofluorescence and confocal imaging. Cells (MCC1 cells transfected with miR-224-5p/inhibitor-224-5p), and corresponding positive and negative control were plated on coverslips or in chamber slides. After reaching a suitable density $\left(8 \times 10^{4} / \mathrm{ml}\right)$, the cells were fixed with $4 \%$ paraformaldehyde overnight at $4{ }^{\circ} \mathrm{C}$, permeabilized in $0.5 \%$ Triton X-100 for $8 \mathrm{~min}$ at room temperature, blocked with phosphate-buffered saline containing $10 \%$ goat serum for $1 \mathrm{~h}$ at room temperature and incubated overnight with anti-LC3 (cat. no. L7543, 1:500 dilution; Sigma-Aldrich; Merck KGaA) at $4^{\circ} \mathrm{C}$. Fixed and stained coverslips or slides were washed 3 times with phosphate-buffered saline, and labeled with Alexa Fluor-conjugated secondary antibodies (cat. no. A11036, 1;200 dilution; Invitrogen; Thermo Fisher Scientific, Inc.). Anti-LC3 antibody was obtained from Sigma-Aldrich; Merck KGaA. The coverslips or slides were mounted with ProLong Gold Antifade Reagent mounting medium containing DAPI (Thermo Fisher Scientific, Inc.), and the cells were visualized by confocal laser microscopy (Olympus Corporation). Three random fields of view of each sample were used for semi-quantification analysis at $60 \mathrm{x}$ magnification. ImageJ software (version $1.44 \mathrm{p}$; National Institutes of Health) was used for semi-quantified analysis.

Immunohistochemical (IHC) analysis. Immunohistochemical (IHC) analysis was performed as previously described (13). The following primary antibodies were used: Anti-LC3 (cat. no. GB11124, 1:500 dilution) antibody and anti-p62 (cat. no. GB11239, 1:500 dilution) antibody from Wuhan Servicebio Technology Co., Ltd. IHC evaluation of protein expression intensity in normal adjacent tissues and paired pancreatic mucinous cystadenocarcinoma tissues was 

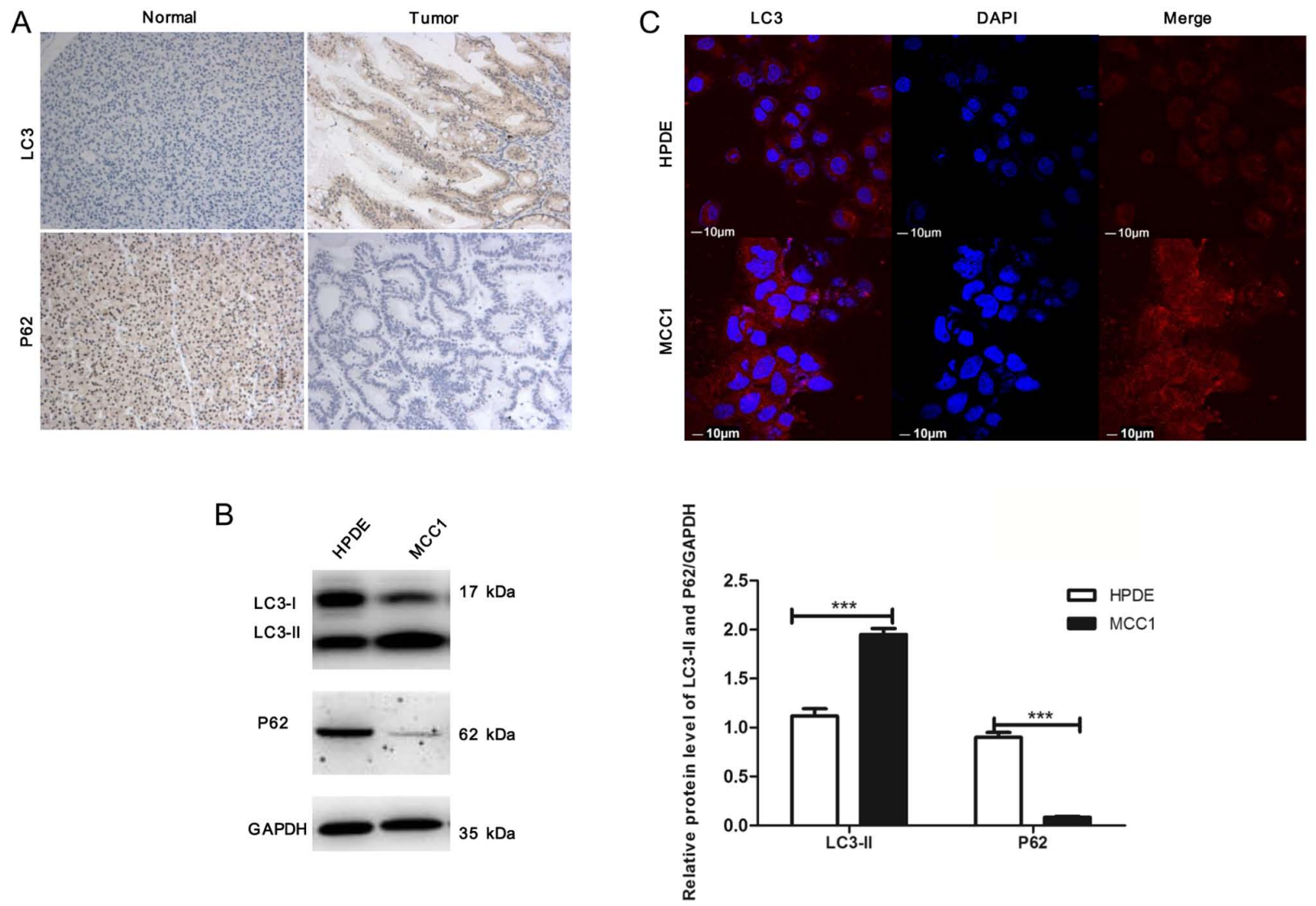

Figure 1. Autophagy is hyperactivated in pancreatic mucinous cystadenocarcinoma tissues and MCC1 cells. (A) Representative immunohistochemistry of pancreatic mucinous cystadenocarcinoma and matched adjacent tissue sections demonstrating the staining of LC3 and P62. Magnification x20. (B) Expression of LC3 and P62 was determined by western blotting in HPDE and MCC1 cells. GAPDH was used as an internal control. (C) Intracellular autophagosome and autolysosomes in HPDE and MCC1 cells were probed by LC3 dots (red) for cell immunofluorescence. Nuclei were stained with DAPI (blue). Scale bar, $10 \mu \mathrm{m}$. Data are presented as the mean $\pm \mathrm{SD}(\mathrm{n}=3)$ from three separate experiments. ${ }^{* * *} \mathrm{P}<0.001$, Student's t-test. Normal, matched adjacent tissue; Tumor, pancreatic mucinous cystadenocarcinoma tissue.

performed independently by two pathologists from the Department of Pathology, Changhai Hospital. Staining intensity was scored as previously described (12). Goat serum (10\%; HyClone; Cyvita) was used in the place of primary antibody as a negative control.

Target gene prediction and luciferase reporter assay. The online tool TargetScan 7.2 (http://www.targetscan.org) was used to predict the miR-224-5p targets. BCL2 was selected as a predicted target of miR-224-5p. A 219-base pair fragment of the BCL2 3'-untranslated region (UTR) sequence containing one putative miR-224-5p binding site was cloned into the pmirGV272-luciferase reporter plasmids (Shanghai GeneChem Co., Ltd.). GV369-miR-224 mimic-transfected MCC1 cells and GV369-miR-224 mimic control-transfected MCC1 cells were transfected with the luciferase reporters using Lipofectamine ${ }^{\circledR}$ 2000. A Thermo Scientific, Inc. Microplate Reader was used to detect firefly luminescence and Renilla luminescence. Results were evaluated through normalization of the firefly luciferase activity with Renilla luciferase activity as previously described (15).

Statistical analysis. Data are presented as the mean \pm SD from at least three independent experiments. SPSS 21.0 software (IBM Corp.) was used to conduct statistical analysis.
Student's t-test was used to compare the differences between two groups; one-way ANOVA followed by the least significant difference post hoc test was used to compare the differences among multiple groups. $\mathrm{P}<0.05$ was considered to indicate a statistically significant difference.

\section{Results}

Autophagy is hyperactivated in pancreatic mucinous cystadenocarcinoma tissues and MCC1. A total of 4 paired pancreatic MCC and adjacent non-tumor tissues were used for IHC staining with anti-LC3 and anti-p62 antibodies. The protein expression of LC3 was higher in the tumors compared with that in adjacent non-tumor tissues, whereas p62 accumulation was decreased in tumor tissues (Fig. 1A). The level of autophagy signaling in MCC1 and HPDE cells was determined by western blotting; the results demonstrated that compared with HPDE cells, the expression levels of LC3-II were significantly increased in MCC1 cells, whereas p62 expression levels were notably decreased (Fig. 1B), which was consistent with the results from clinical samples. Additionally, immunofluorescence (IF) was performed to detect autophagosomes, which revealed that the number of LC3 dots (red) in MCC1 cells was increased compared with HPDE cells (Fig. 1C). These results indicated that autophagy 
A

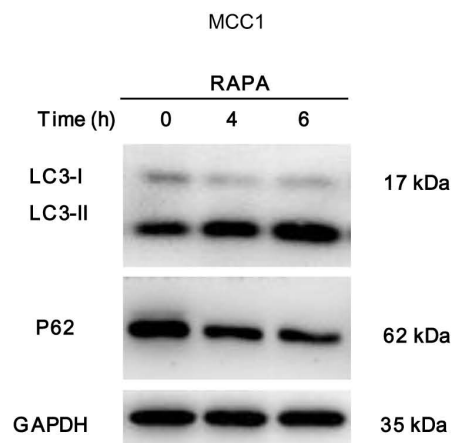

B
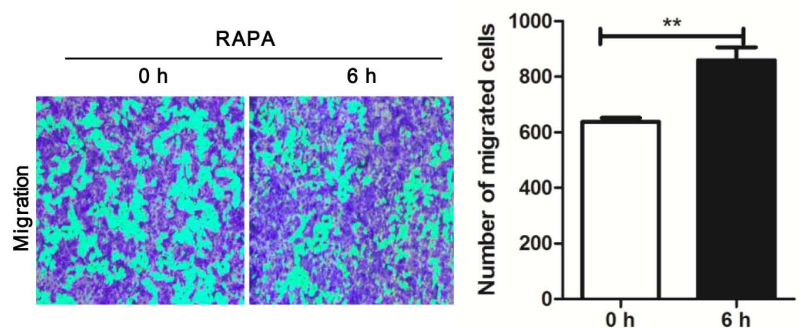

D
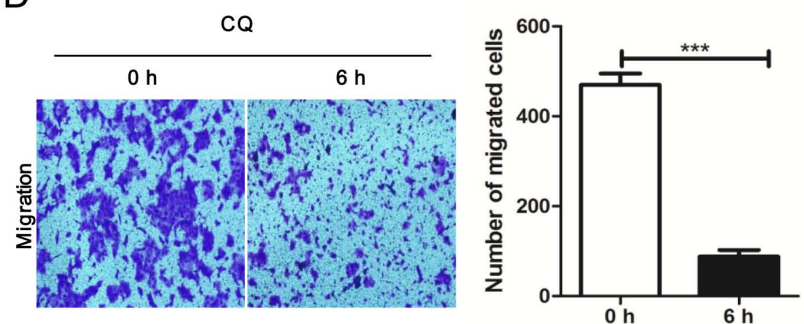

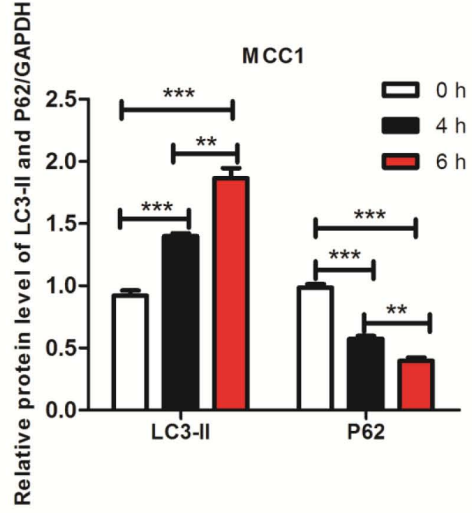

C
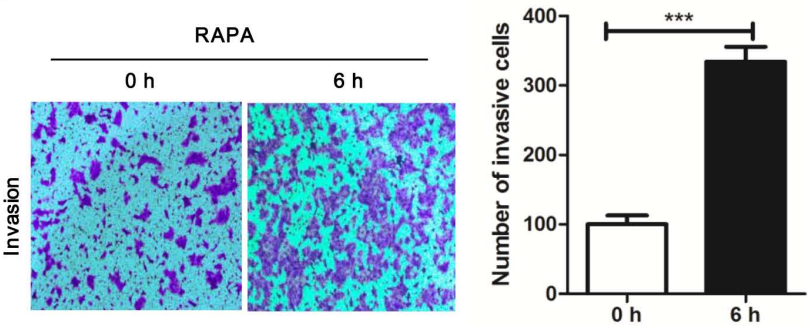

E
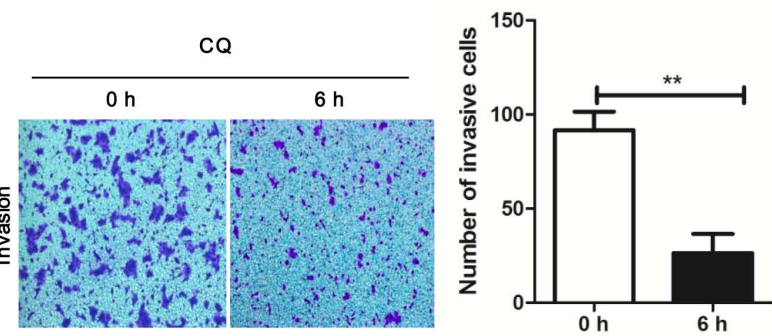

Figure 2. Autophagy promotes the migration and invasion of MCC1. (A) MCC1 cells were treated with $100 \mu \mathrm{M}$ rapamycin for 0 h, 4 h or 6 h. LC3 and P62 expression was detected by western blot analysis. Relative protein expression levels of LC3-II and P62 were normalized to GAPDH. (B) Migration (magnification x20) and (C) invasion (magnification x20) Transwell assays were conducted using MCC1 cells stimulated with $100 \mu \mathrm{M}$ rapamycin for 6 h. Quantitative results are presented. (D) Migration (magnification x20) and (E) invasion (magnification x20) Transwell assays were conducted using MCC1 cells stimulated with $100 \mu \mathrm{M}$ CQ for $6 \mathrm{~h}$. Quantitative results are presented. Data are presented as the mean $\pm \mathrm{SD}(\mathrm{n}=3)$ from three separate experiments. ${ }^{* *} \mathrm{P}<0.01$ and ${ }^{* * *} \mathrm{P}<0.001$, Student's t-test. RAPA, rapamycin; CQ, Chloroquine; miR, microRNA. Fold represents the relative ratio of migrated/invaded cells.

was hyperactivated in pancreatic MCC tissues and MCC1 cells.

Autophagy promotes the migration and invasion of $\mathrm{MCCl}$ cells. To investigate the potential effects of autophagy on the migration and invasion of MCC1 cells, the mTOR inhibitor rapamycin was used to treat $\mathrm{MCC} 1$ for 0,4 or $6 \mathrm{~h}$. The results demonstrated that rapamycin induced changes in the expression of proteins associated with autophagy activity (Fig. 2A). In addition, the results of the Transwell assay revealed that the migration and invasion of rapamycin-treated MCC1 cells was significantly enhanced compared with that in the control groups (Fig. 2B and C). Additionally, the migration and invasion of MCC1 cells treated with CQ was significantly decreased compared with that in the control groups (Fig. 2D and E). These results demonstrated that autophagy facilitated the migration and invasion of MCC1 cells.

miR-224-5p promotes autophagy in MCCl cells. The present study examined whether miR-224-5p expression levels changed in response to stress-induced stimuli in MCC1. To verify this hypothesis, MCC1 cells were treated with rapamycin to induce autophagy; the results demonstrated that miR-224-5p expression levels increased in a time-dependent manner (Fig. 3A). Additionally, miR-224-5p expression levels were significantly increased in MCC1 cells compared with those in HPDE cells (Fig. 3B). Therefore, these results suggested an association between autophagy activation and miR-224-5p levels in $\mathrm{MCC} 1$ cells (Figs. 1B and 3B).

The present study examined whether miR-224-5p affected autophagy in MCC1 cells. Lentiviruses with a miR-224-5p mimic or miR-224-5p inhibitor were constructed to interfere with the level of miR-224-5p in MCC1 cells, and RT-qPCR assay was performed to assess the expression levels of miR-224-5p in MCC1. The results revealed overexpression of miR-224-5p in the miR-224-5p mimic group compared with that in the control group (Fig. 3C). Western blot analysis demonstrated that compared with the negative control, the expression of LC3-II was upregulated, whereas that of p62 was reduced in MCC1 cells overexpressing miR-224-5p (Fig. 3E). In addition, 
A

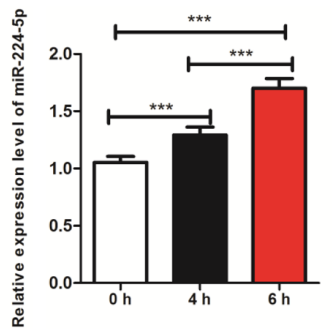

C

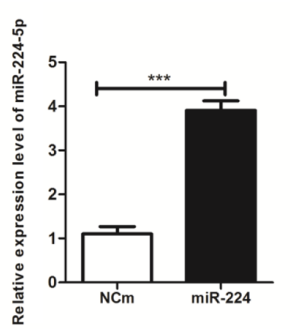

G
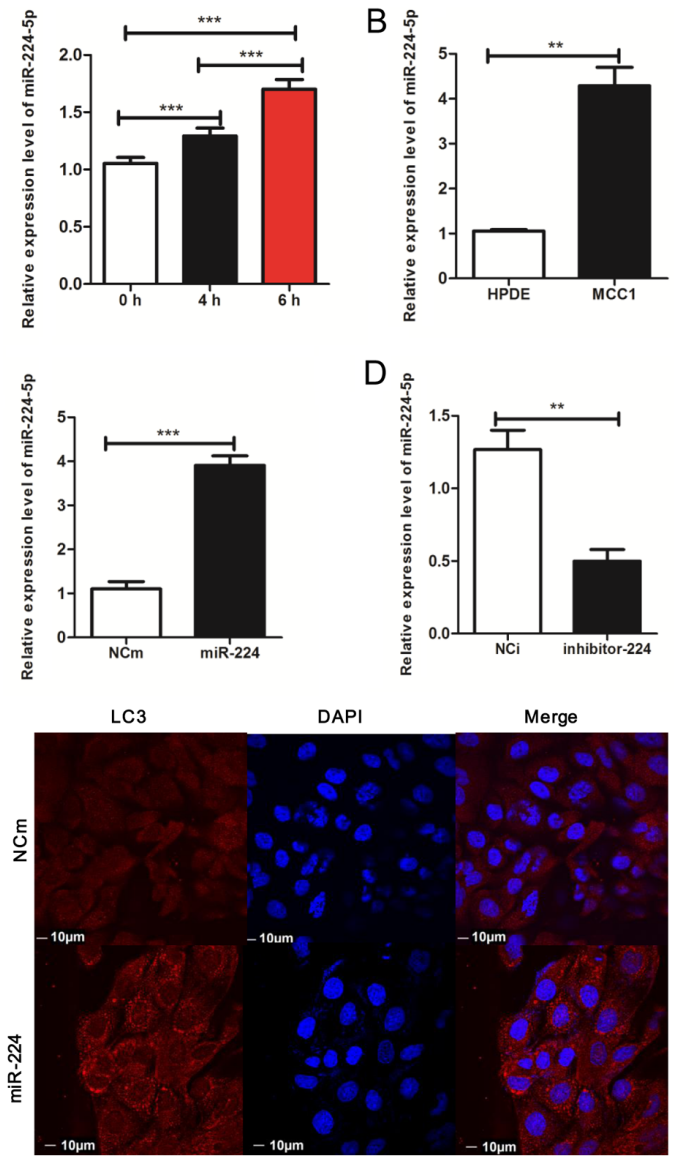

$\mathrm{D}$ 。ั

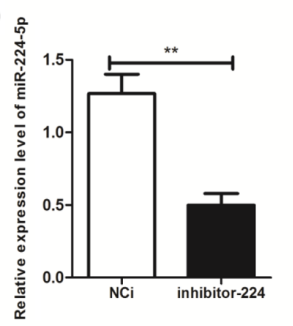

$\mathrm{E}$

$\mathrm{F}$
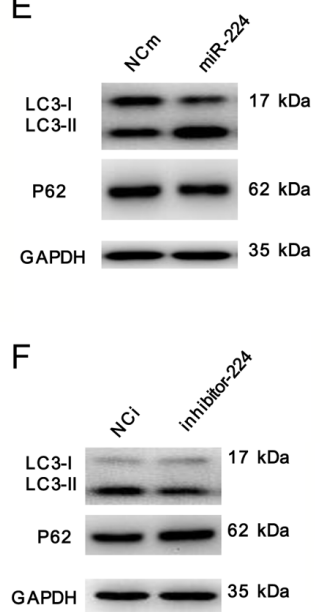

GAPDH

$\mathrm{H}$

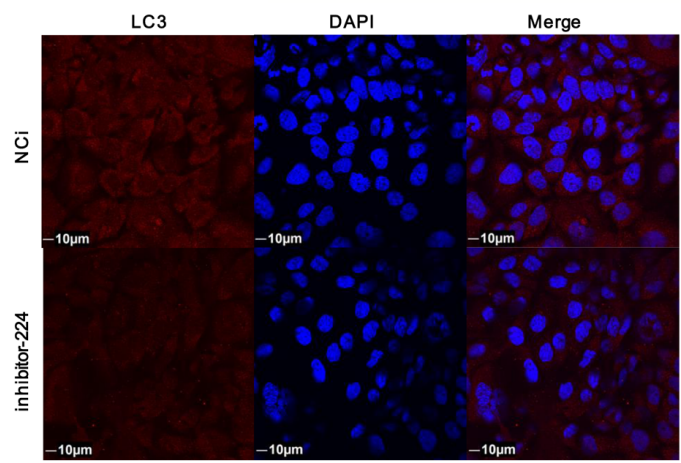

Figure 3. miR-224-5p promotes autophagy in MCC1 cells. (A) miR-224-5p expression in cells was measured by Reverse transcription-quantitative PCR (B) miR-224-5p expression in MCC1 and HPDE cells was measured by reverse transcription-quantitative PCR. (C) Expression levels of miR-224-5p in cells treated with miR-224-5p mimic and miR-224-5p mimic control. (D) Expression levels of miR-224-5p in cells treated with miR-224-5p inhibitor and miR-224-5p inhibitor control. (E and F) Effects of (E) miR-224-5p mimic and (F) miR-224-5p inhibitor on autophagy of lentivirus transduced MCC1 cells. Expression of LC3 and p62 was detected by western blot analysis. Relative protein expression of LC3-II and p62 were normalized to GAPDH. (G and H) The results of immunofluorescence suggested that $(\mathrm{G})$ overexpression or $(\mathrm{H})$ knockdown of miR-224-5p affected the number of LC3 fluorescent dots (red) in MCC1 cells, indicating autophagosome formation. Nuclei were stained with DAPI (blue). Scale bar, $10 \mu \mathrm{m}$. Data are presented as the mean \pm SD ( $=3$ ) from three separate experiments. ${ }^{*} \mathrm{P}<0.05,{ }^{* *} \mathrm{P}<0.01$ and ${ }^{* * * *} \mathrm{P}<0.001$, Student's t-test. miR, microRNA; NCm, miR-224-5p mimic control; miR-224, miR-224-5p mimic; NCi, miR-224-5p inhibitor control; inhibitor-224, miR-224-5p inhibitor.

overexpression of miR-224-5p increased the number of fluorescent LC3 dots in MCC1 cells compared with that in the negative control (Fig. 3G), which indicated the accumulation of autophagosomes. By contrast, miR-224-5p was downregulated in the presence of the miR-224-5p inhibitor compared with the negative control group (Fig. 3D). Additionally, the loss of miR-224-5p inhibited the expression of LC3II and elevated the expression of p62 compared with that in the negative control (Fig. 3F). The results of the IF assay confirmed that the inhibition of miR-224-5p expression repressed autophagosome accumulation in MCC1 cells compared with the negative control (Fig. 3H). Overall, these results demonstrated that autophagy significantly increased the expression level of miR-224-5p in MCC1 cells and that miR-224-5p may facilitate autophagy in the pancreatic mucinous cystadenocarcinoma cell line MCC1, suggesting that positive feedback may occur between autophagy and miR-224-5p in MCC1.

miR-224-5p promotes autophagy through BCL2 in MCC1. To explore the molecular mechanisms of miR-224-5p-mediated autophagy, TargetScan Human 7.2 was used to screen potential downstream targets of miR-224-5p in autophagy;
BCL2 was identified as a target gene (Fig. 4A). To confirm whether BCL2 was a direct target of miR-224-5p, a luciferase reporter assay was performed. The results demonstrated that overexpression of miR-224-5p reduced luciferase activity in the BCL2 wild-type 3'-UTR reporter, but had no effect on the BCL2 mutant 3'-UTR reporter (Fig. 4B). These results suggested that BCL2 may be a direct target of miR-224-5p. In addition, the levels of BCL2 were evaluated in $\mathrm{MCC} 1$ cells overexpressing miR-224-5p, which revealed that the miR-224-5p mimic significantly decreased the mRNA and protein levels of BCL2 (Fig. 4C and D). Consistently, inhibition of miR-224-5p increased BCL2 expression at the mRNA and protein level (Fig. 4E and F). To verify the effects of BCL2 on miR-224-5p-induced autophagy, MCC1 cells were transfected with siBCL2, which abolished the expression of BCL2 (Fig. 4G). BCL2 silencing significantly rescued autophagy in cells transfected with the miR-224 inhibitor, demonstrating that BCL2 protein knockdown counteracted the effects of the miR-224-5p inhibitor on autophagy inhibition (Fig. 4H). The expression levels of BCL2 were also determined in MCC1 and HPDE cells by western blotting; the results demonstrated that compared with HPDE cells, the expression levels of BCL2 
A

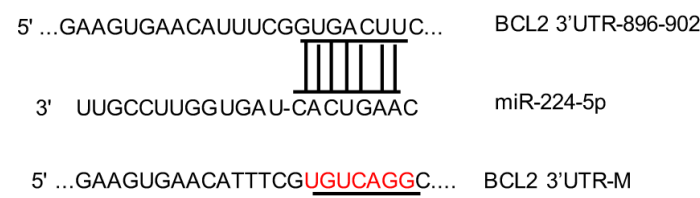

B

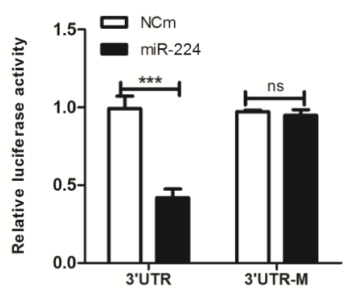

C

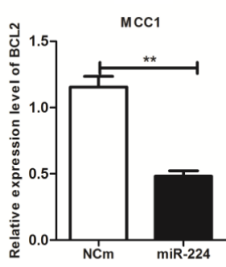

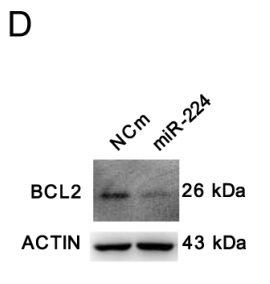

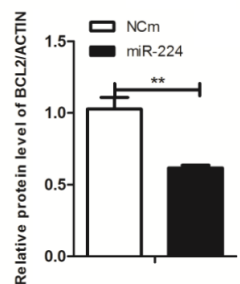

E

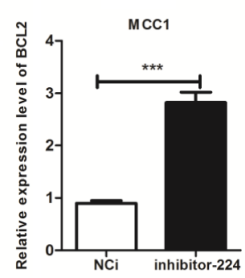

$\mathrm{F}$
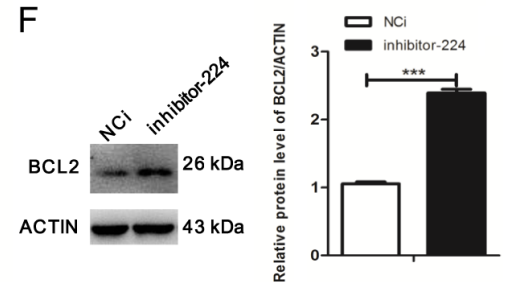

G

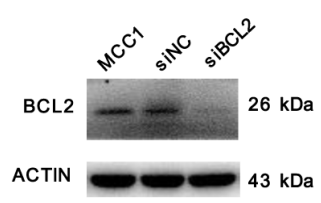

$\mathrm{H}$

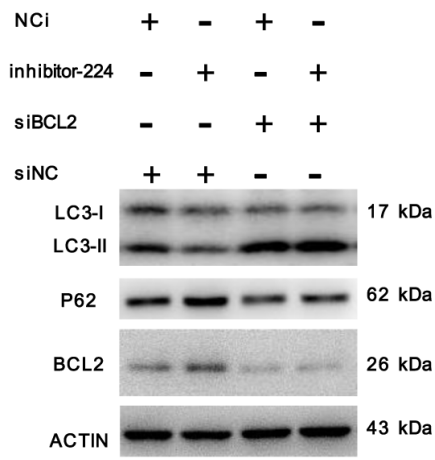

I
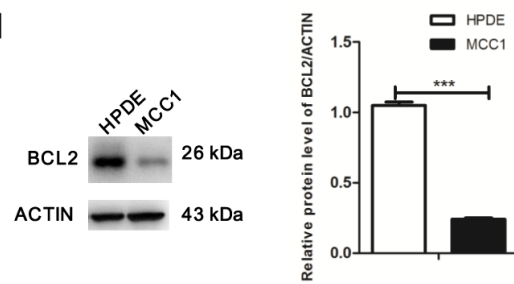

Tumor

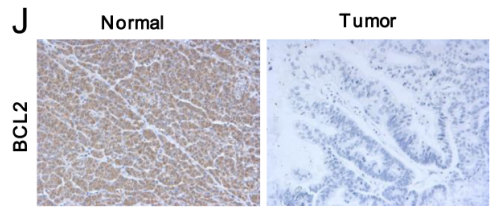

Figure 4. miR-224-5p promotes autophagy through BCL2 in MCC1 cells. (A) Predicted binding sequences for miR-224-5p in the BCL2 3'-UTR. (B) Luciferase reporter vectors containing wild-type or mutated 3'-UTR fragments of BCL2 cloned into pmirGV272-luciferase plasmids. Luciferase activity was assayed $48 \mathrm{~h}$ following co-transfection with either wild-type or mutated 3'-UTR containing plasmids and miR-224-5p mimic or miR-224-5p mimic control in MCC1 cells. (C) Overexpression of miR-224-5p affected the expression level of BCL2 in MCC1 determined by reverse transcription-quantitative PCR. (D) Overexpression of miR-224-5p affected the protein expression level of BCL2 in MCC1 cells. Relative protein expression of BCL2 was normalized to the level of $\beta$-actin. (E) Inhibition of miR-224-5p affected the mRNA expression level of BCL2 in MCC1 cells. (F) Inhibition of miR-224-5p affected the protein expression level of BCL2 in MCC1 cells. Relative protein expression of BCL2 was normalized to $\beta$-actin. (G) MCC1 cells were transfected with BCL2 siRNA. Western blotting was used to detect the expression of BCL2. (H) miR-224-5p inhibitor and miR-224-5p inhibitor control-treated MCC1 cells were transfected with BCL2 siRNA, and western blot analysis was performed to detect the expression levels of LC3, P62 and BCL2. (I) Expression of BCL2 was determined by western blotting in HPDE and MCC1 cells. Actin was used as an internal control. (J) Representative immunohistochemistry images of pancreatic mucinous cystadenocarcinoma and matched adjacent tissue sections demonstrating the staining of BCL2 (magnification x20). Data are presented as the mean \pm SD $(\mathrm{n}=3)$ from three separate experiments. ${ }^{* *} \mathrm{P}<0.01$ and ${ }^{* * *} \mathrm{P}<0.001$, Student's t-test. miR, microRNA; NCm, miR-224-5p mimic control; miR-224, miR-224-5p mimic; NCi, miR-224-5p inhibitor control; inhibitor-224, miR-224-5p inhibitor; siNC, siBCL2 control; UTR, untranslated region; M, mutated; siRNA, small interfering RNA; Normal, matched adjacent tissue; Tumor, pancreatic mucinous cystadenocarcinoma tissue.

were reduced in $\mathrm{MCCl}$ cells (Fig. 4I). The protein expression of BCL2 was decreased in tumor tissues compared with that in adjacent non-tumor tissues (Fig. 4J). These results suggested that miR-224-5p may functionally promote autophagy through BCL2.

\section{Discussion}

Although autophagy serves an important role in cancer (16), the function of autophagy in regulating pancreatic MCC is unknown. The results of the present study demonstrated that autophagy was increased in pancreatic MCC tissues and cells compared with the respective control groups and promoted the migration and invasion of the pancreatic mucinous cystadenocarcinoma cell line MCC1. In addition, the expression levels of miR-224-5p were significantly increased in MCC1 cells when autophagy was activated. A previous study has suggested that BCL2 inhibits autophagy by directly interacting with the
BH3 domain of beclin 1 in the endoplasmic reticulum (17). Further experiments demonstrated that miR-224-5p facilitated autophagy in MCC1 by directly targeting BCL2. These results suggested that a positive feedback loop between autophagy and miR-224-5p may promote the migration and invasion of MCC1 cells.

Metastasis is one of the leading causes of cancer-related death. The present study demonstrated that autophagy promoted the migration and invasion of MCC1 cells. The association between autophagy and tumors has been studied previously; findings have suggested that autophagy also influences tumor metastasis. Autophagy promotes the invasion of the mutant KRAS-transformed human breast cancer cell line MDA-MB-231 by secreting the cytokine interleukin- 6 (18), and autophagy inhibition substantially reduces tumor metastasis in mice (19). Previous studies have also suggested that the role of autophagy in tumor metastasis is multifaceted and depends on the cell type, tumor microenvironment and genomic 
status $(11,20)$. Autophagy inhibits metastasis by blocking the epithelial-mesenchymal transition and fibrosis, but promotes metastasis by promoting local adhesion, anti-anoikis and coupling of tumor interstitial metabolism (11). Defects in autophagy can promote the development of pancreatitis and thus affect the risk of pancreatic cancer $(21,22)$. Additionally, a previous study has demonstrated that continuously activated autophagy can lead to autophagy-induced death of pancreatic cancer cells, thus, inhibiting tumorigenesis (23). These findings suggested that the variable effects of autophagy on the development of pancreatic cancer may be associated with the stage at which it is detected. By IHC analysis of pancreatic cancer tissues of 71 patients who underwent pancreaticoduodenectomy, compared with adjacent non-tumor tissue, autophagy activity in pancreatic cancer tissue was increased compared with adjacent non-tumor tissue and significantly associated with poor prognosis (24). Overexpression and constitutive activation of the microphthalmia/transcription factor $\mathrm{E}$ family transcription factors in pancreatic cancer further mediate lysosomal amplification and hyperactivate autophagy to promote the development of pancreatic cancer (25). The results of the present study demonstrated that autophagy promoted the migration and invasion of MCC1 cells, supporting a role for autophagy in the metastasis of pancreatic mucinous cystadenocarcinoma. It is speculated that inhibition of autophagy may be a potential therapeutic strategy for pancreatic mucinous cystadenocarcinoma. Considering the crucial role of autophagy in tumor metabolism and immunity $(26,27)$, the effects of autophagy on metabolism and tumor immunity in pancreatic mucinous cystadenocarcinoma will be investigated further.

Previous studies have suggested that autophagy regulates miRNA expression or activity $(28,29)$. Inhibition of autophagy leads to the inhibition of miRNA function (30). In our previous study, miR-224 was decreased in pancreatic mucinous cystadenoma (31), whereas the results of the present study revealed that miR-224-5p was significantly increased in MCC1, which appears contradictory. Compared with pancreatic duct epithelial cells in a normal pancreas, mild and moderate pancreatic intraepithelial neoplasia, moderate or severe LC3 staining was identified in $81 \%$ of pancreatic duct epithelial cells and the pancreatic cancer cell cytoplasm with severe intraepithelial neoplasia (32), suggesting that the autophagic activity of tumor cells is related to tumor stages; the autophagy activity increased with tumor progression. Pancreatic mucinous cystadenoma and cystadenocarcinoma are different stages of tumor progression. However, autophagy serves an important role in regulating miRNAs and the relatively high autophagy activation also upregulates the expression of miR-224-5p in MCC1. miRNAs have been widely reported to be involved in the process of autophagy (33-36). The results of the present study revealed that miR-224-5p may facilitate autophagy in MCC1.

In conclusion, the results of the present study demonstrated that autophagy promoted the migration and invasion of pancreatic mucinous cystadenocarcinoma MCC1 cells and that autophagy elevated the expression levels of miR-224-5p in MCC1. Additionally, miR-224-5p promoted autophagy by targeting BCL2. These results suggested that pancreatic mucinous cystadenocarcinoma may exhibit hyperactivated autophagy. The results also revealed that an autophagy/miR-224-5p positive feedback loop may promote the migration and invasion of $\mathrm{MCC} 1$ cells and provided insight into the association between autophagy and tumor metastasis in pancreatic mucinous cystadenocarcinoma.

\section{Acknowledgements}

The authors would like to thank Professor Claudio Sorio (University of Verona, Italy) for the MCC1 cell line.

\section{Funding}

The present study was supported by grants from the National Key R\&D Program of China (grant no. 2017YFC1600100), the Strategic Priority Research Program of the Chinese Academy of Sciences (grant no. XDA12010100) and the National Natural Science Foundation of China (grant no. 81672892).

\section{Availability of data and materials}

The datasets used and/or analyzed during the current study are available from the corresponding author on reasonable request.

\section{Authors' contributions}

$\mathrm{XZ}$ and $\mathrm{LZ}$ conceived the study. XP and $\mathrm{CG}$ acquired the data. LS and YW analyzed the data. XZ acquired funding. CG, LS and JL performed the experiments. RC and DF developed the methodology. CG supervised the study. CG, MY and XP analyzed the data and interpreted the results. $C G$ wrote the manuscript. $\mathrm{XZ}$ and $\mathrm{LZ}$ revised the manuscript.

\section{Ethics approval and consent to participate}

The present study was approved by the Shanghai Changhai Hospital Ethics Committee (Shanghai, China) (approval no. C HEC2016-8167111578). Informed consent was obtained from the subjects prior to the collection of the specimens.

\section{Patient consent for publication}

Not applicable.

\section{Competing interests}

The authors declare that they have no competing interests.

\section{References}

1. Farrell JJ: Pancreatic cysts and guidelines. Dig Dis Sci 62: $1827-1839,2017$

2. Canto MI and Hruban RH: Managing pancreatic cysts: Less is more? Gastroenterology 148: 688-691, 2015.

3. Compagno J and Oertel JE: Mucinous cystic neoplasms of the pancreas with overt and latent malignancy (cystadenocarcinoma and cystadenoma). A clinicopathologic study of 41 cases. Am J Clin Pathol 69: 573-580, 1978.

4. Postlewait LM, Ethun CG, McInnis MR, Merchant N, Parikh A Idrees K, Isom CA, Hawkins W, Fields RC, Strand M, et al: Association of preoperative risk factors with malignancy in pancreatic mucinous cystic neoplasms: A multicenter study. JAMA Surg 152: 19-25, 2016. 
5. Sarr MG, Carpenter HA, Prabhakar LP, Orchard TF, Hughes S, van Heerden JA and DiMagno EP: Clinical and pathologic correlation of 84 mucinous cystic neoplasms of the pancreas: Can one reliably differentiate benign from malignant (or premalignant) neoplasms? Ann Surg 231: 205-212, 2000.

6. Del Chiaro M, Verbeke C, Salvia R, Klöppel G, Werner J, McKay C, Friess H, Manfredi R, Van Cutsem E, Löhr M, et al: European experts consensus statement on cystic tumours of the pancreas. Dig Liver Dis 45: 703-711, 2013.

7. Tanaka M,Fernández-del Castillo C, Adsay V, Chari S, Falconi M, Jang JY, Kimura W, Levy P, Pitman MB, Schmidt CM, et al International consensus guidelines 2012 for the management of IPMN and MCN of the pancreas. Pancreatology 12: 183-197, 2012

8. Mizushima N: A brief history of autophagy from cell biology to physiology and disease. Nat Cell Biol 20: 521-527, 2018.

9. Kim J, Kundu M, Viollet B and Guan KL: AMPK and mTOR regulate autophagy through direct phosphorylation of ulk1. Nat Cell Biol 13: 132-141, 2011.

10. Levy JM, Towers CG and Thorburn A: Targeting autophagy in cancer. Nat Rev Cancer 17: 528-542, 2017.

11. Dower CM, Wills CA, Frisch SM and Wang HG: Mechanisms and context underlying the role of autophagy in cancer metastasis. Autophagy 14: 1110-1128, 2018.

12. Xu Y, Chang R, Peng Z, Wang Y, Ji W, Guo J, Song L, Dai C, Wei $\mathrm{W}, \mathrm{Wu} \mathrm{Y}$, et al: Loss of polarity protein AF6 promotes pancreatic cancer metastasis by inducing snail expression. Nat Commun 26: 7184, 2015

13. Chang R, Song L, Xu Y, Wu Y, Dai C, Wang X, Sun X, Hou Y, Li W, Zhan X and Zhan L: Loss of Wwox drives metastasis in triple-negative breast cancer by JAK2/STAT3 axis. Nat Commun 28: 3486, 2018.

14. Livak KJ and Schmittgen TD: Analysis of relative gene expression data using real-time quantitative PCR and the 2(-Delta Delta C(T)) method. Methods 25: 402-408, 2001.

15. Xu XD, Song XW, Li Q, Wang GK, Jing Q and Qin YW: Attenuation of microRNA-22 derepressed PTEN to effectively protect rat cardiomyocytes from hypertrophy. J Cell Physiol 227: 1391-1398, 2012

16. Maes H, Rubio N, Garg AD and Agostinis P: Autophagy: Shaping the tumor microenvironment and therapeutic response Trends Mol Med 19: 428-446, 2013.

17. $\mathrm{He} \mathrm{C}$ and Levine B: The beclin 1 interactome. Curr Opin Cell Biol 22: 140-149, 2010.

18. Lock R, Kenific CM, Leidal AM, Salas E and Debnath J: Autophagy-dependent production of secreted factors facilitates oncogenic RAS-driven invasion. Cancer Discov 4: 466-479, 2014

19. Sharifi MN, Mowers EE, Drake LE, Collier C, Chen H, Zamora M, Mui S and Macleod KF: Autophagy promotes focal adhesion disassembly and cell motility of metastatic tumor cells through the direct interaction of paxillin with LC3. Cell Rep 15 1660-1672, 2016.

20. Görgülü K, Diakopoulos KN, Ai J, Schoeps B, Kabacaoglu D, Karpathaki AF, Ciecielski KJ, Kaya-Aksoy E, Ruess DA, Berninger A, et al: Levels of the autophagy-related 5 protein affect progression and metastasis of pancreatic tumors in mice. Gastroenterology 156: 203-217, 2019.

21. Diakopoulos KN, Lesina M, Wörmann S, Song L, Aichler M, Schild L, Artati A, Römisch-Margl W, Wartmann T, Fischer R, et al: Impaired autophagy induces chronic atrophic pancreatitis in mice via sex- and nutrition-dependent processes. Gastroenterology 148: 626-638, 2015.
22. Antonucci L, Fagman JB, Kim JY, Todoric J, Gukovsky I, Mackey M, Ellisman MH and Karin M: Basal autophagy maintains pancreatic acinar cell homeostasis and protein synthesis and prevents ER stress. Proc Natl Acad Sci USA 112: E6166-E6174, 2015.

23. Sun L, Hu L, Cogdell D, Lu L, Gao C, Tian W, Zhang Z, Kang Y, Fleming JB and Zhang W: MIR506 induces autophagy-related cell death in pancreatic cancer cells by targeting the STAT3 pathway. Autophagy 13: 703-714, 2017.

24. Fujii S, Mitsunaga S, Yamazaki M, Hasebe T, Ishii G, Kojima M, Kinoshita T, Ueno T, Esumi H and Ochiai A: Autophagy is activated in pancreatic cancer cells and correlates with poor patient outcome. Cancer Sci 99: 1813-1819, 2008.

25. Perera RM, Stoykova S, Nicolay BN, Ross KN, Fitamant J, Boukhali M, Lengrand J, Deshpande V, Selig MK, Ferrone CR, et al: Transcriptional control of autophagy-lysosome function drives pancreatic cancer metabolism. Nature 524: 361-365, 2015.

26. Kimmelman AC and White E: Autophagy and tumor metabolism. Cell Metab 25: 1037-1043, 2017.

27. Rybstein MD, Bravo-San Pedro JM, Kroemer G and Galluzzi L: The autophagic network and cancer. Nat Cell Biol 20: 243-251, 2018.

28. Lan SH, Wu SY, Zuchini R, Lin XZ, Su IJ, Tsai TF, Lin YJ, Wu CT and Liu HS: Autophagy suppresses tumorigenesis of hepatitis B virus-associated hepatocellular carcinoma through degradation of microRNA-224. Hepatology 59: 505-517, 2014.

29. Jing Z, Han W, Sui X, Xie J and Pan H: Interaction of autophagy with microRNAs and their potential therapeutic implications in human cancers. Cancer Lett 356: 332-338, 2015.

30. Gibbings D, Mostow S, Jay F, Schwab Y, Cossart P and Voinnet O Selective autophagy degrades DICER and AGO2 and regulates miRNA activity. Nat Cell Biol 14: 1314-1321, 2012.

31. Zhang B, Guo X,Zhang J,Liu X,Zhan X and Li Z: MicroRNA-224 is downregulated in mucinous cystic neoplasms of the pancreas and may regulate tumorigenesis by targeting jagged1. Mol Med Rep 10: 3303-3309, 2014.

32. Yang S, Wang X, Contino G, Liesa M, Sahin E, Ying H, Bause A, Li Y, Stommel JM, Dell'antonio G, et al: Pancreatic cancers require autophagy for tumor growth. Genes Dev 25: 717-729, 2011.

33. Füllgrabe J, Klionsky DJ and Joseph B: The return of the nucleus: Transcriptional and epigenetic control of autophagy. Nat Rev Mol Cell Biol 15: 65-74, 2014.

34. Li Y, Jiang J, Liu W, Wang H, Zhao L, Liu S, Li P, Zhang S, Sun $\mathrm{C}, \mathrm{Wu} \mathrm{Y}$, et al: MicroRNA-378 promotes autophagy and inhibits apoptosis in skeletal muscle. Proc Natl Acad Sci USA 115: E10849-E10858, 2018.

35. Li Z, Wang G, Feng D, Zu G, Li Y, Shi X, Zhao Y, Jing H, Ning S, Le W, et al: Targeting the miR-665-3p-ATG4B-autophagy axis relieves inflammation and apoptosis in intestinal ischemia/reperfusion. Cell Death Dis 9: 483.2018.

36. Korkmaz G, le Sage C, Tekirdag KA, Agami R and Gozuacik D: MiR-376b controls starvation and mTOR inhibition-related autophagy by targeting ATG4C and BECN1. Autophagy 8: $165-176,2012$.

This work is licensed under a Creative Commons Attribution-NonCommercial-NoDerivatives 4.0 International (CC BY-NC-ND 4.0) License. 